Portland State University

PDXScholar

\title{
Toll-like Receptor 7 Expression and Function on Sensory Nerves
}

Karol Wai

Portland State University

Follow this and additional works at: https://pdxscholar.library.pdx.edu/honorstheses Let us know how access to this document benefits you.

\section{Recommended Citation}

Wai, Karol, "Toll-like Receptor 7 Expression and Function on Sensory Nerves" (2018). University Honors Theses. Paper 627.

https://doi.org/10.15760/honors.605

This Thesis is brought to you for free and open access. It has been accepted for inclusion in University Honors Theses by an authorized administrator of PDXScholar. Please contact us if we can make this document more accessible: pdxscholar@pdx.edu. 
Toll-like Receptor 7 Expression and Function on Sensory Nerves

by

Karol Wai

An undergraduate honors thesis submitted in partial fulfillment of the requirements for the degree of

Bachelor of Science

in

University Honors

and

Biology

Thesis Adviser

Matthew G. Drake

Portland State University 


\begin{abstract}
Neuronal Toll-like Receptor 7 Regulates Airway Sensory Nerve Growth

Karol Wai, Becky J. Proskocil, Emily D. Blum, Katherine M. Lebold, Allison D. Fryer, David B. Jacoby, Matthew G. Drake.
\end{abstract}

Background: Toll-like receptor 7 (TLR7) is an innate immune receptor that detects singlestranded RNA viruses and triggers an immune response. Recently, we found that airway sensory nerves express TLR7, although its role on nerves is unknown.

Objective: To characterize TLR7 expression on airway sensory nerves, and to determine the effects of neuronal TLR7 stimulation.

Methods: Vagal and dorsal root ganglia were isolated from female Hartley guinea pigs ( 400g) and fixed in zinc formalin. Nerves were immunolabeled with antibodies against TLR7 and either neurofilament-1 (NF-H) to identify $\mathrm{A} \delta$ fiber sensory nerves or transient receptor potential V1 (TRPV1) to identify $\mathrm{C}$ fiber sensory nerves. In separate experiments, dorsal root ganglia were isolated, cultured, and treated with the TLR7 agonist R837 (0.1-100 microM) for 16 hours. Nerve length, the number of neurites, and the number of branch points were quantified.

Results: Airway sensory nerves originating in the vagal and dorsal root ganglia heterogeneously expressed TLR7. TLR7 was highly expressed on small TRPV1-expressing C fiber neurons, but not on large NF-H-positive A $\delta$ neurons. The TLR7 agonist R837 dose-dependently increased nerve length and branching in vitro.

Conclusions: TLR7 is highly expressed by airway sensory $\mathrm{C}$ fibers nerves and its activation stimulates neurite growth. Neuronal TLR7 may facilitate detection of respiratory viruses and regulate anti-viral immune responses. 
Funding: NIH grants HL121254, HL 124165, HL113023, AR061567, ES017592, UL1GM118964, and TL4GM118965 


\section{INTRODUCTION}

\section{Innervation to the Lung}

Sensory afferent nerve cell bodies reside in clusters (ganglia) and project nerve fibers to the airway epithelium. These fibers primarily originate in the vagal ganglia located at the base of the skull (21), which are divided into nodose and jugular ganglia (10). A smaller population of airway sensory neurons originate in the dorsal root ganglia located along the thoracic spine (20). Sensory nerves detect irritants including cigarette smoke, mechanical stimuli, changes in $\mathrm{pH}$ or temperature, allergens, and pollutants in the airway lumen and relay signals to the central nervous system (CNS) $(11,12,13,14,15)$. Upon transmission of the signals by sensory afferent nerves, the CNS then stimulates the efferent parasympathetic cholinergic nerves that mediate physiological effects such as cough and bronchoconstriction (narrowing of the airways) (16).

\section{A $\delta$ and C Sensory Nerve Fibers}

Sensory nerves are composed of different types of fibers. Slow, non-myelinated C fibers are responsible for the perception of long lasting pain, and fast, myelinated A $\delta$ fibers are responsible for the detection of sharp pain triggering a withdrawal reflex $(4,5)$. Signals detected as "slow" pain come from the stimulation and transmission of nerve impulses over C fibers, while "fast" pain comes from stimulation and transmission over $\mathrm{A} \delta$ fibers. A $\delta$ fiber neurons have myelin sheaths composed of proteins and lipids, enabling faster conduction velocities. In comparison, C fiber neurons have slower conduction velocities less than $1.3 \mathrm{~m} / \mathrm{s}$ attributed to the lack of myelin and small diameters. The intensity of immunoreactivity to a neurofilament- 1 antibody and cell size (cross-sectional area) have been used to distinguish $\mathrm{A} \delta$ fibers from $\mathrm{C}$ fibers. A $\delta$ fiber cell bodies are neurofilament-1 positive and larger in size whereas $\mathrm{C}$ fiber cell bodies are neurofilament-1 negative and smaller in size (6). 


\section{Nerve Growth and Branching}

Vertebrate neurons integrate information from different regions. This is enabled by the ability of a single nerve fiber (axon) to extend numerous branches and form terminal arbors on target tissue. Nerve branches originate from the axon shaft and arborize on specific targets. Growth factors such as fibroblast growth factor (FGF), and neurotrophins such as nerve growth factor (NGF) and brain derived neurotrophic factor (BDNF) contribute to neurite branching and arborization (19). Changes in arborization and innervation significantly alter physiological responses in the airways. Studies have shown that overexpression of NGF in transgenic mice result in local hyperinnervation in tissues that expressed the transgene (22). Hyperinnervation increases sensitivity to capsaicininduced airway constriction detected by sensory afferent $\mathrm{C}$ fibers (22) and magnifies physiological responses. On the contrary, the loss of nerves lessen physiological responses. 
Fig.1.1. Nerve Overview-parasympathetic nerves and sensory nerve pathways (adapted from reference 33).

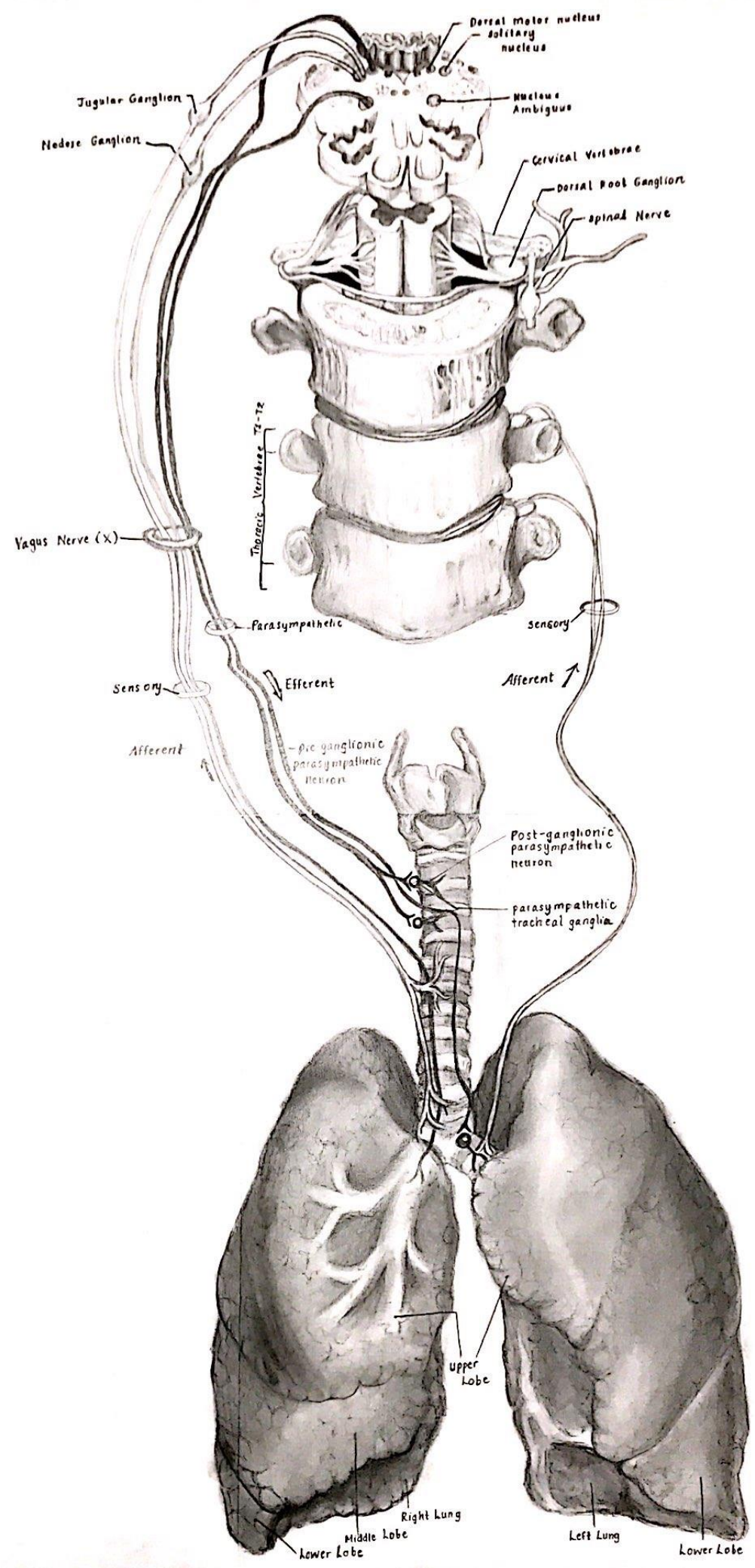




\section{TLR7 Expression and Activation}

Toll-like Receptor 7 (TLR7) is a single stranded RNA receptor located within intracellular vesicles like endosomes or lysosomes and the endoplasmic reticulum of eukaryotic cells (1). It belongs to the Toll-like Receptor (TLR) family of innate immune receptors, which play a critical role in host defense by detecting pathogen-associated molecular patterns including lipids, proteins, and nucleic acids from bacteria, funguses, parasites, and viruses $(23,24)$. The TLRs responsible for recognizing viral products predominantly do so by detecting virus nucleic acids. TLR7 specifically detects virus single-stranded RNA (ssRNA) and resides on airway epithelial cells where binding of respiratory virus triggers an innate immune response by producing type 1 interferons and inflammatory cytokines (2). Binding of respiratory virus ssRNA to TLR7 not only initiates the inflammatory and immune response, but also worsens asthma (25) and induces airway hyperreactivity $(26,27)$. Stimulation of TLR7 with R837 agonist has also been shown to rapidly dilate guinea pig airways in vivo (28) as well as human airways in vitro (3). These findings, combined with the immunomodulatory abilities of TLR7 to suppress airway hyperreactivity common after virus infections in asthma (30) and prevent Th2-mediated airway inflammation (31, 32), heighten its potential as a therapeutic target in asthma. In this study, we investigate the expression and role of TLR7 on airway sensory nerves. 


\section{METHODS}

\section{Animals}

Female Hartley guinea pigs ( 400 g) were purchased from Charles River Labs, Wilmington, MA and were handled according to the U.S. Animal Welfare Protocols approved by the Institutional Animal Care and Use Committee. Guinea pigs are an ideal model for these experiments since human and guinea pig airway have similar airway innervation and physiology.

\section{Immunohistochemistry}

Vagal and dorsal root ganglia were isolated from female Hartley guinea pigs ( $400 \mathrm{~g})$ and fixed in zinc formalin overnight. Ganglia were processed for paraffin embedding and then sectioned. Paraffin slides were placed in xylene overnight. Slides were de-waxed in xylene and then rehydrated in series of ethanol $(100 \%, 70 \%, 50 \%)$ to distilled water. Antigens were unmasked by $1 \%$ antigen unmasking solution (AUS; Vector) diluted in distilled water in a $90^{\circ} \mathrm{C}$ water bath. Following additional water washes, ganglia were blocked in $10 \%$ normal goat serum (NGS) and phosphate-buffered saline solution with $0.05 \%$ tween (PBST) at room temperature for one hour. The ganglia were immunolabeled with antibodies against TLR7 (Abcam/ Novus) and either neurofilament-1 (NF-H; Abcam) to identify A $\delta$ fibers or transient receptor potential V1 (TRPV1; Alomone) to identify C fibers. Primary antibodies were diluted in PBST containing $10 \%$ NGS and slides were kept in a humidified chamber overnight at $4^{\circ} \mathrm{C}$. Control slides were not treated with primary antibodies (no primary control). Slides were then warmed up to room temperature and protected from light during secondary antibody application (see below). Slides were covered with vectashield with DAPI to elucidate the nuclei (Vector H-1200), sealed with Cytoseal (Thermo Scientific 8310-16), and stored flat at $4^{\circ} \mathrm{C}$ until imaging. 
Dorsal root ganglia were immunolabeled as follows:

- Rabbit polyclonal antibody against TLR7 (Abcam)

- Goat anti-rabbit 488 F(ab')2 -conjugated antibody (LifeTech)

- Chicken polyclonal antibody against Neurofilament-1 (Abcam)

- Goat anti-chicken 555 F(ab')2 -conjugated antibody (LifeTech)

Vagal ganglia were immunolabeled as follows when using TRPV1 as a C fiber marker:

- Rabbit polyclonal antibody against TLR7 (Novus)

- Goat anti-rabbit 488 (LifeTech A11034; Alexa Flour 488 IgG H+L)

- Rabbit polyclonal antibody against TRPV1 (Alomone ACC-030)

- Goat anti-rabbit 555 (LifeTech A21429; Alexa Fluor 555 IgG H+L)

Vagal ganglia were immunolabeled as follows when using Neurofilament-1 (NF-H) as an A $\delta$ fiber marker:

- Rabbit polyclonal antibody against TLR7 (Novus)

- Goat anti-rabbit 488 (LifeTech A11034; Alexa Flour 488 IgG H+L)

- Chicken polyclonal antibody against Neurofilament-1 (Abcam)

- Goat anti-chicken 555 (Invitrogen A21437; Alexa Fluor 555 IgG H+L)

\section{Microscopy}

Ganglia were photographed under identical conditions using an ApoTome confocal microscope (20X, 1.3 NA). Four images were taken per nodose and jugular ganglia while one image was taken for each whole dorsal root ganglia. Thirteen dorsal root ganglia were analyzed from 2 animals. Ten nodose ganglia and nine jugular ganglia were analyzed from five animals. Data obtained from each ganglia was averaged per animal. Pictures were taken at the wavelengths 488 (to visualize TLR7), 555 (to visualize TRPV1 or NF-H), and 405 (DAPI). 


\section{Image J/ FIJI software}

FIJI software was used to measure the fluorescent intensity and cross-sectional area of each neuron within the dorsal root, nodose, and jugular ganglia. Neurons were defined as enclosed cell bodies having a nucleus and were traced using the FIJI drawing tool. The fluorescent intensity of NF-H, TRPV1, and TLR7 was recorded for each neuron as well as the area of each neuron. Values from the background and no primary control tissues were subtracted from the mean fluorescent intensities of the fully stained tissues. The fluorescence indicated the level of brightness within each nerve cell and was used to quantify expression. A threshold was established for each image and it enabled clear delineations between TLR7-positive neurons and TLR7-negative neurons based on their fluorescence. Neurons were then categorized as either TLR7-positive or TLR7negative depending on whether or not they significantly expressed TLR7. 
Figure 1.2. A) Dorsal root ganglion (5X). TLR7 (green), NF-H (red), DAPI (blue)). B) Whole dorsal root ganglion split into DAPI (blue), TLR7 (green), and NF-H (red) channels. C) A magnified image showing traced neurons inversely express NF-H (red) and TLR7 (green).
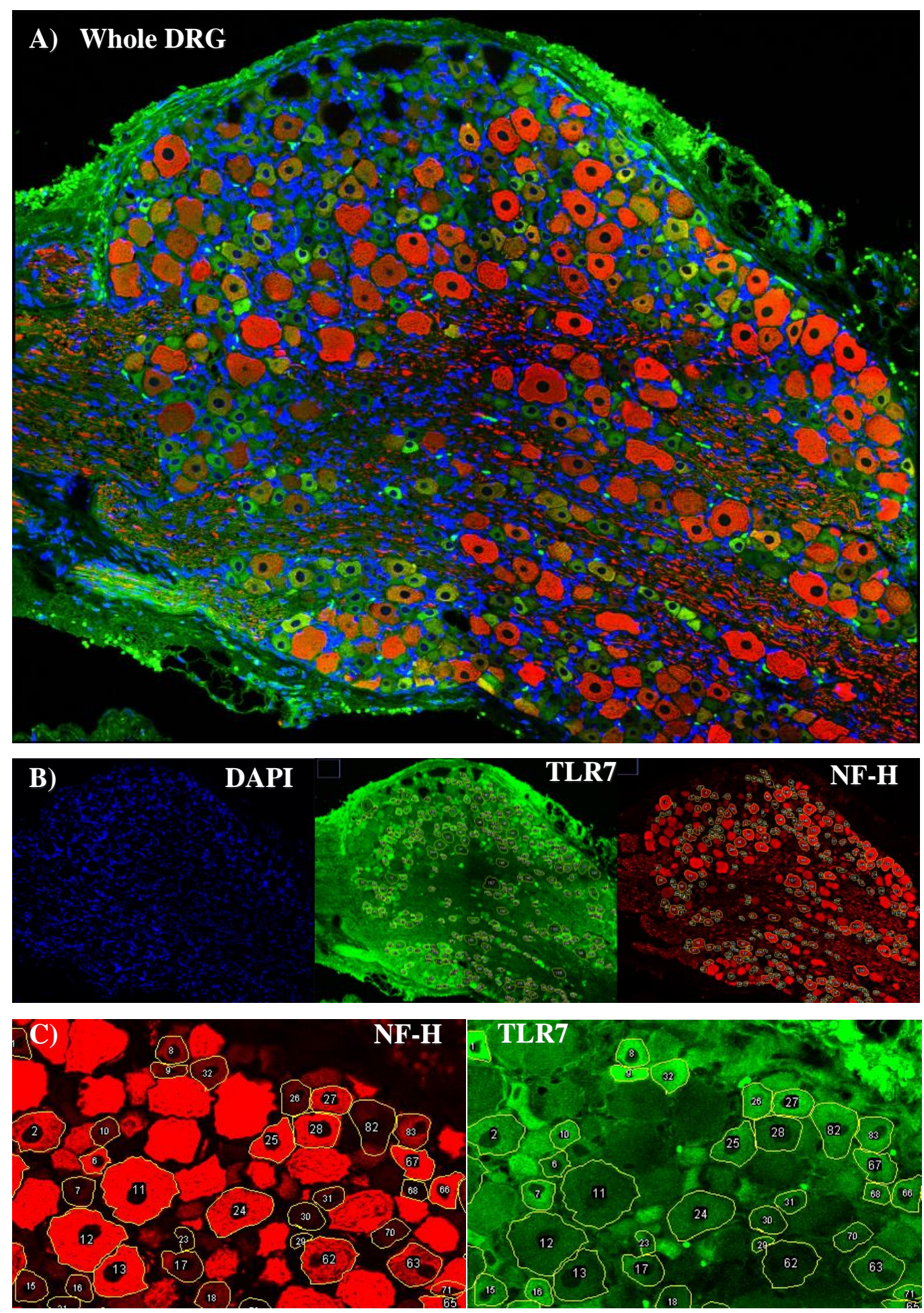
Figure 1.3. A) Nodose Ganglia (5x). B) Nodose Ganglia No Primary Control (5x). TLR7 (green), NF-H (red), DAPI (blue).

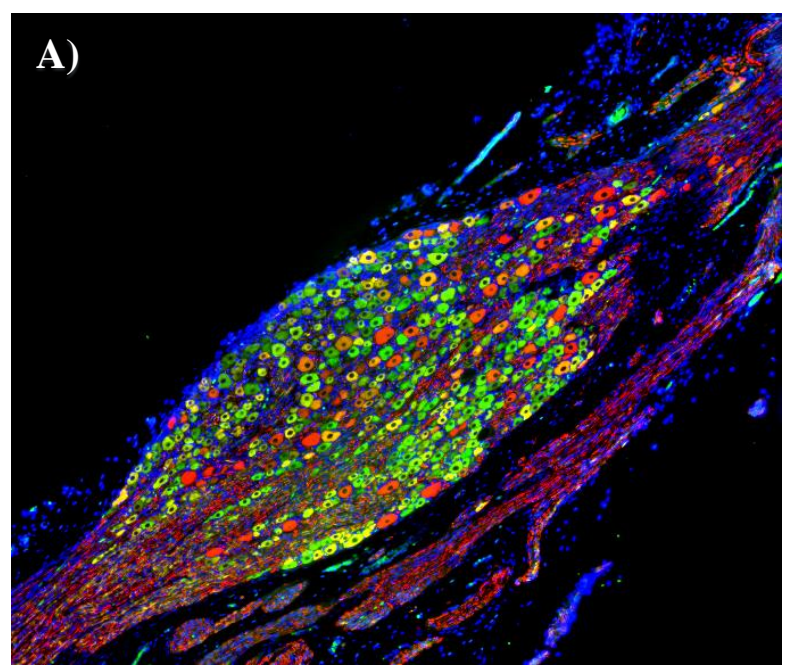

B)

Figure 1.4. A) Jugular Ganglia (5x). B) Whole Jugular Ganglia No Primary Control (5x). TLR7 (green), NF-H (red), DAPI (blue).
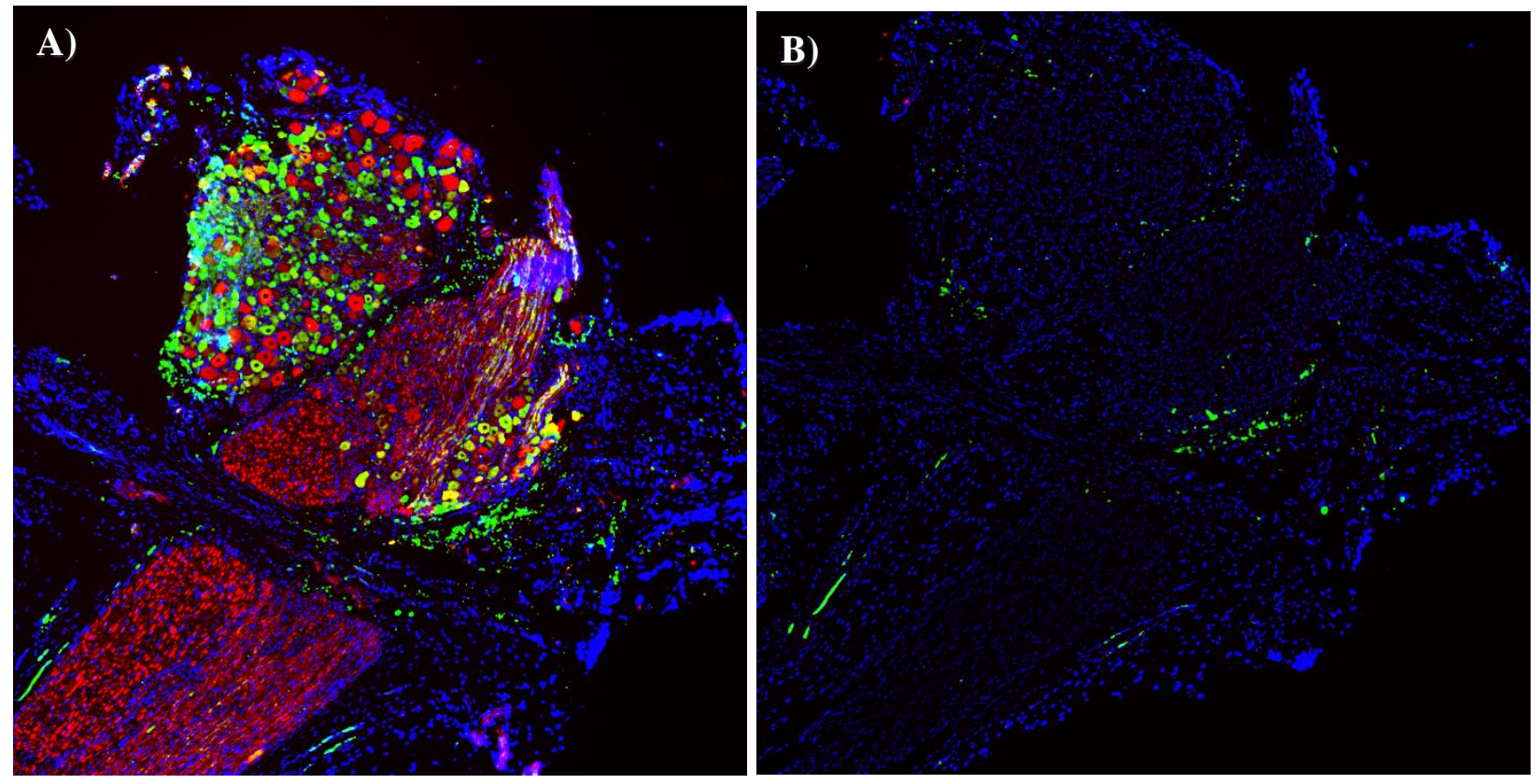


\section{Nerve Culture}

Dorsal root ganglia were isolated and digested in $600 \mathrm{U} / \mathrm{mL}$ type IV collagenase for 5 minutes at $37^{\circ} \mathrm{C}$ while gently agitated and cultured in serum-free media containing nerve growth factor (100 $\mu \mathrm{g} / \mathrm{mL}$; Harlan). Nerve cultures were treated with TLR7 agonist R837 (0.1-10 $\mu \mathrm{M})$ for 16 hours then fixed in zinc formalin. Nerve images were obtained with a Nikon Eclipse TS100 microscope (10X magnification). Nerve length was measured by a blinded observer using FIJI software. All experimental conditions were conducted in triplicate and total $\mathrm{n}=3$.

\section{Statistics}

The percentage and cross-sectional area of NF-H and TRPV1 expressing neurons that were TLR7negative or TLR7-positive neurons were analyzed using a two-tailed, unpaired T-test (GraphPad Software, La Jolla, CA). Changes in nerve length were analyzed using a one-way ANOVA with the Bonferroni post-hoc test. Data is represented as mean \pm SEM. A $p$ value less than 0.05 was considered significant. 


\section{RESULTS}

Dorsal root and vagal sensory nerves contain more TLR7-positive neurons than TLR7negative neurons.

The percentage of TLR7-negative and TLR7-positive neurons was quantified for guinea pig dorsal root and vagal ganglia. More TLR7-positive neurons than TLR7-negative neurons were present in each ganglia. Specifically, within the dorsal root, nodose, and jugular ganglia, the percentage of TLR7-negative neurons was 32\%, 35\%, and 30\%, while the percentage of TLR7-positive neurons was 68,65 , and $70 \%$ respectively (Figure 2.1 ).

TLR7-positive neurons in the dorsal root and vagal ganglia are smaller than the TLR7negative neurons.

TLR7-positive neurons were smaller on average (based on 2-dimensional cross-sectional area) than TLR7-negative neurons (Figure 2.2). Smaller cells are consistent with unmyelinated C fibers in ganglia suggesting TLR7 is preferentially expressed on this neuronal population.

\section{TLR7-positve neurons express less NF-H than TLR7-negative neurons.}

Guinea pig dorsal root and vagal ganglia were co-stained for TLR7 and NF-H (A $\delta$ fiber marker). Minimal neurofilament-1 expression was detected in TLR7-positive neurons, while high neurofilament-1 expression was observed in TLR7-negative neurons (Figure 3.1). Therefore, TLR7 is primarily expressed by neurons other than A $\delta$ fibers. 
TRPV1 Expression is greater in TLR7-positive neurons than TLR7-negative neurons

Guinea pig vagal ganglia were co-stained for TLR7 and TRPV1. High TRPV1 expression distinguishes small size unmyelinated $\mathrm{C}$ from large myelinated A $\delta$ fibers $(8,9)$. Greater TRPV1 expression was observed in TLR7-positive neurons while low TRPV1 expression was observed in TLR7-negative neurons of vagal ganglia (Figure 3.2). Since high TRPV1 expression is associated with unmyelinated $\mathrm{C}$ fibers, my results indicate that TLR7 is preferentially expressed on sensory C fibers.

\section{TLR7 agonist promotes nerve growth in vitro}

Guinea pig dorsal root ganglia were isolated, cultured, and treated with TLR7 agonist R837 at varying concentrations (100 $\mathrm{nM}, 1 \mu \mathrm{M}$, and $10 \mu \mathrm{M})$ for 16 hours) to determine the effects of neuronal TLR7 stimulation. R837 increased the nerve length in a dose-dependent manner (Figure 4), suggesting TLR7 regulates nerve growth. 
Figure 2.1. There are a greater percentage of TLR7-positive neurons (green bars) than TLR7-negative neurons (white bars) within A) dorsal root ganglia B) nodose ganglia and C) jugular ganglia.

\section{A) Dorsal Root Ganglia}

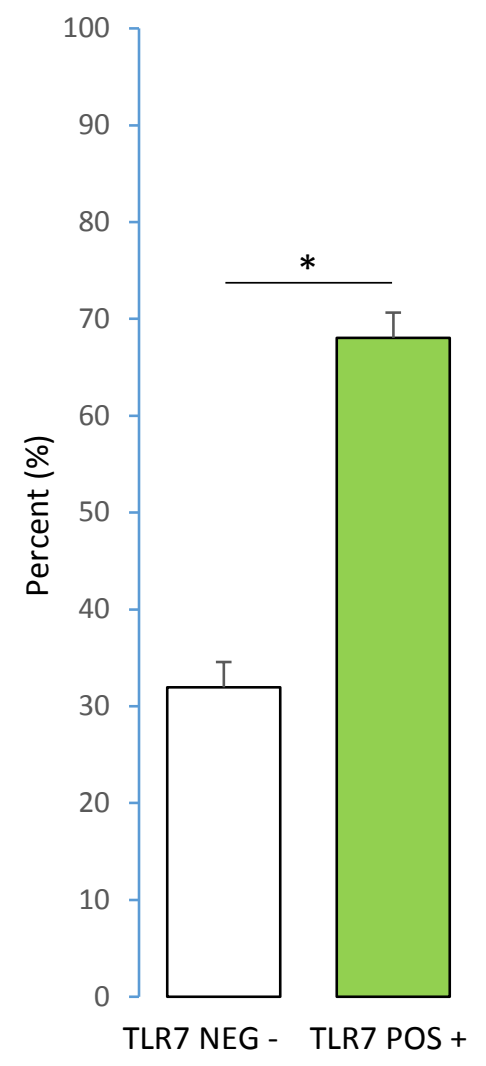

B) Nodose Ganglia

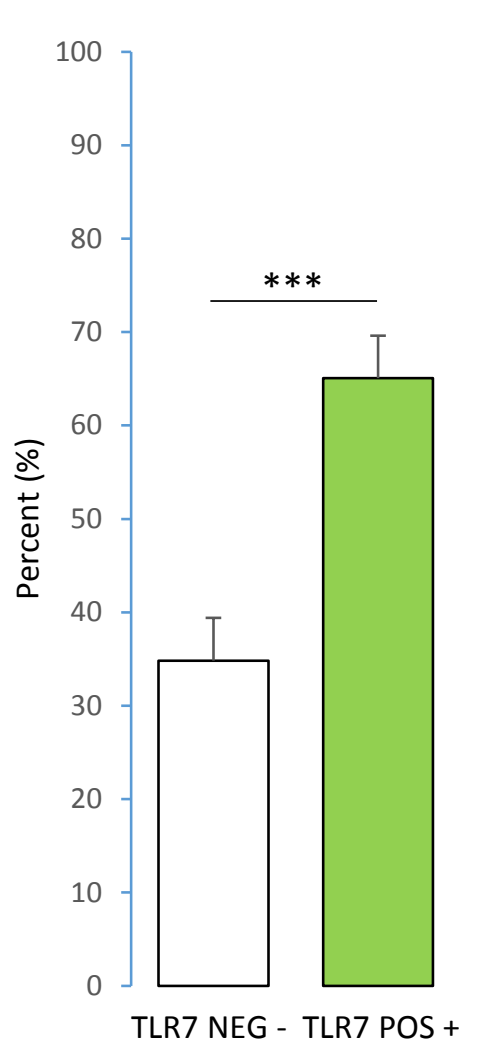

C) Jugular Ganglia

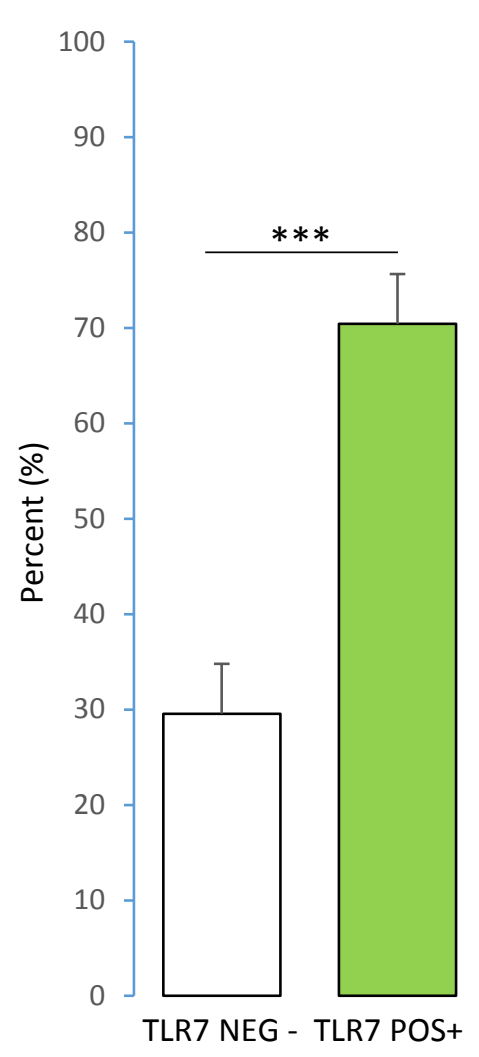

Figure 2.1. The percentage of TLR7-positive and TLR7-negative neurons was calculated within dorsal root and vagal ganglia. Data are expressed as the mean \pm SEM. $(n=2, P=0.0203$ for dorsal root ganglia; $\mathrm{n}=10, \mathrm{P}=0.0003$ for nodose ganglia; $\mathrm{n}=9, \mathrm{P} \leq 0.0001$ for jugular ganglia) 
Figure 2.2. TLR7-negative neurons (white bars) have larger cross-sectional area than TLR7positive neurons (green bars) within A) dorsal root ganglia B) nodose ganglia and C) jugular ganglia.

A) Dorsal Root Ganglia

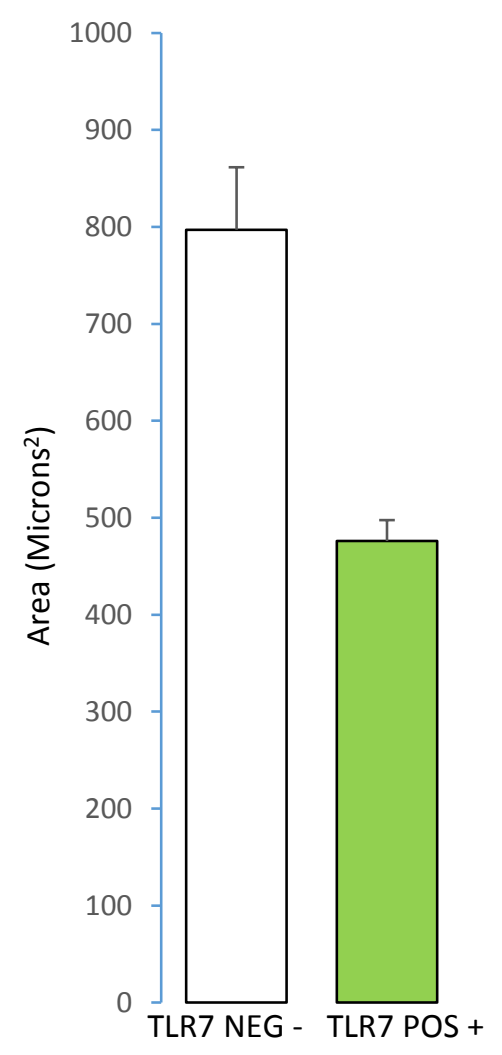

B) Nodose Ganglia

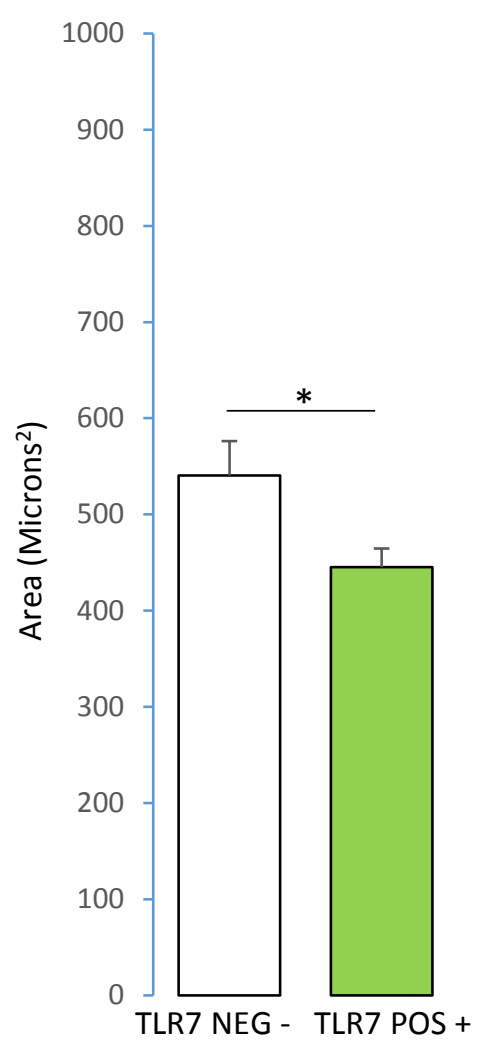

C) Jugular Ganglia

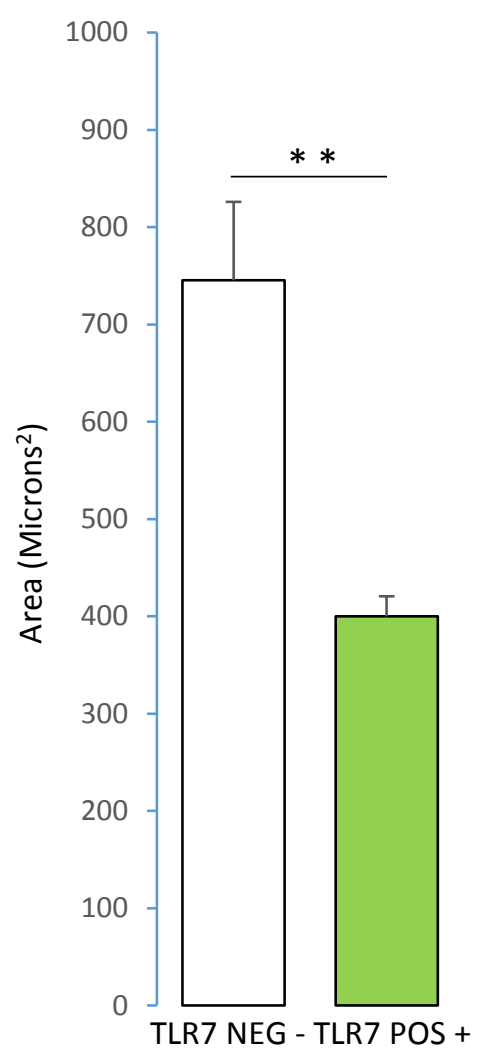

Figure 2.2. The cross-sectional area of TLR7-positive and TLR7-negative neurons was measured. Data are expressed as the mean \pm SEM. $(n=2, P=0.0791$ for dorsal root ganglia; $n=10, P=0.039$ for nodose ganglia; $\mathrm{n}=9, \mathrm{P}=0.0012$ for jugular ganglia) 
Figure 3.1. NF-H expression is greater in TLR7-negative neurons (white bars) than TLR7positive neurons (green bars) within A) dorsal root ganglia B) nodose ganglia and C) jugular ganglia.

A) Dorsal Root Ganglia

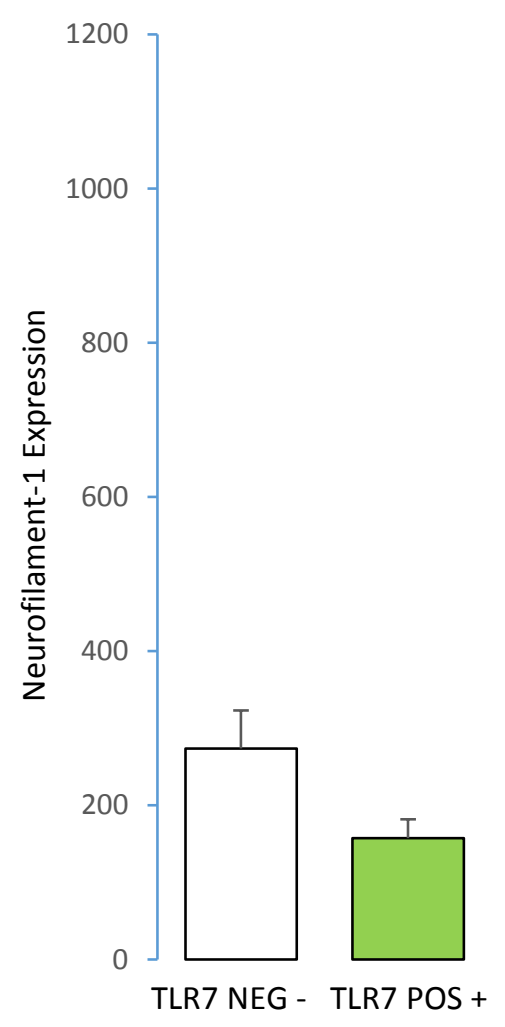

B) Nodose Ganglia

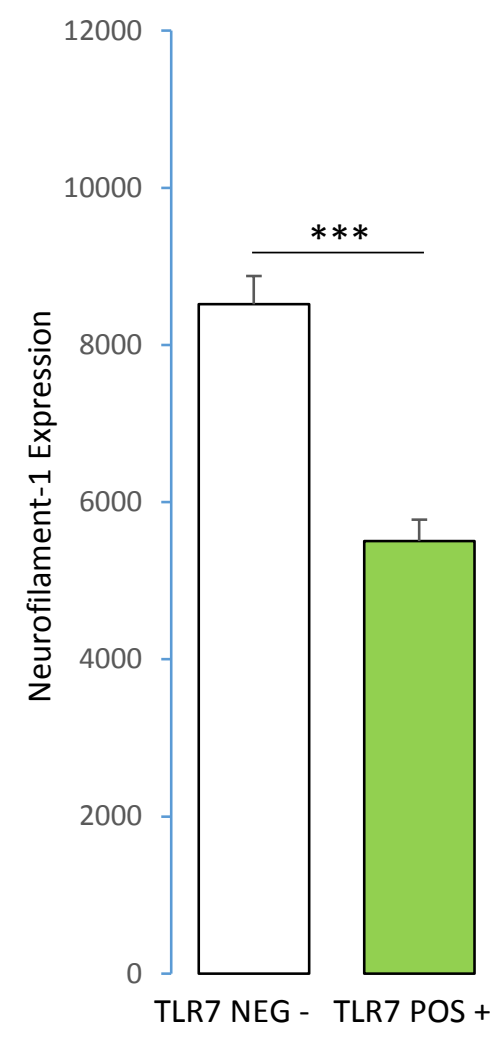

C) Jugular Ganglia

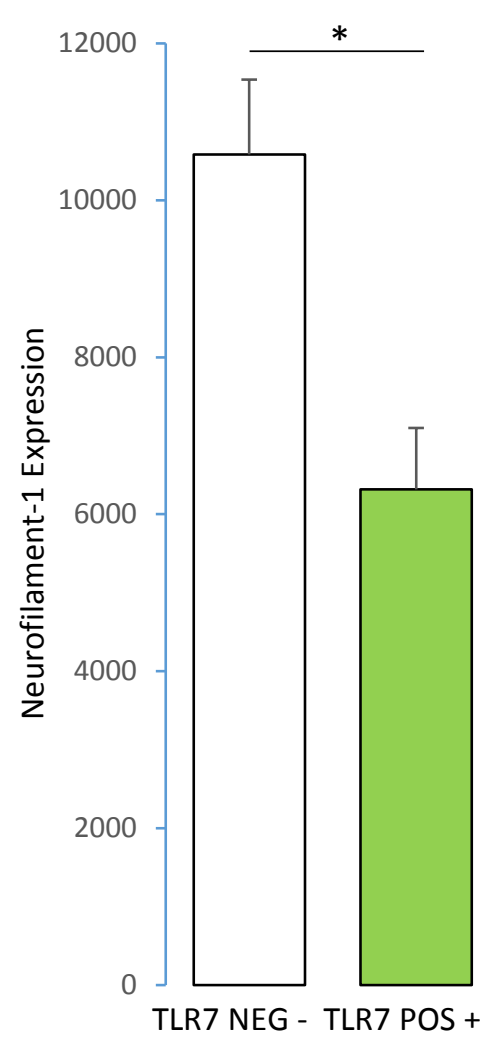

Figure 3.1. Dorsal root and vagal ganglia were immunolabeled for TLR7 and NF-H to determine TLR7 expression on A $\delta$ sensory nerves. Data are expressed as the mean \pm SEM. $(n=2, P=0.2733$ for dorsal root ganglia; $\mathrm{n}=5, \mathrm{P}=0.0003$ for nodose ganglia; $\mathrm{n}=5, \mathrm{P}=0.0147$ for jugular ganglia). 
Figure 3.2. TRPV1 expression is greater in TLR7-positive neurons (green bars) than TLR7negative neurons (white bars) within A) nodose and B) jugular ganglia.

\section{A) Nodose Ganglia}

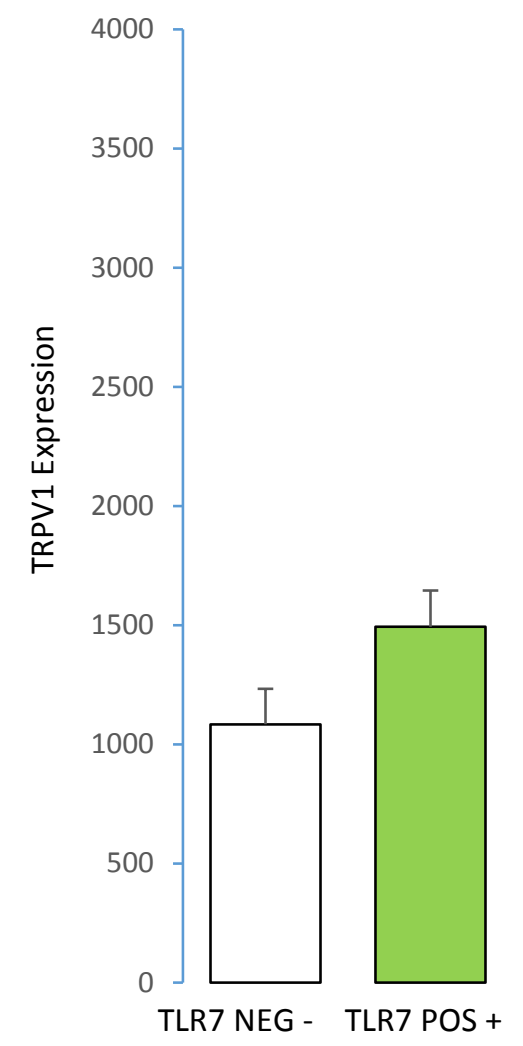

\section{B) Jugular Ganglia}

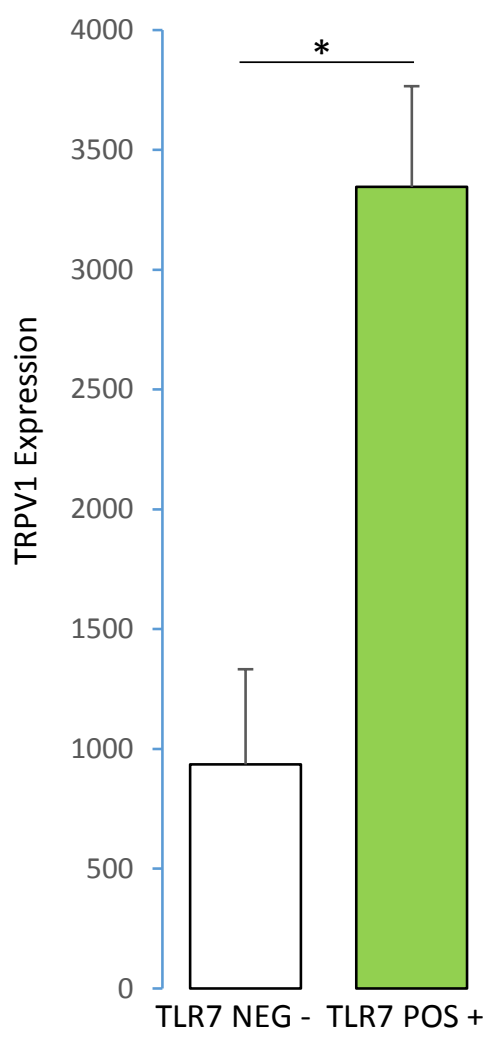

Figure 3.2. Vagal ganglia were immunolabeled for TLR7 and TRPV1 to determine TLR7 expression on C fibers. Greater TRPV1 expression was observed in TLR7-positive neurons while less TRPV1 expression was observed in TLR7-negative neurons in vagal ganglia. Data are expressed as the mean \pm SEM. $(n=5, P=0.1234$ for nodose ganglia; $n=4, P=0.0112$ for jugular ganglia). 
Figure 4: TLR7 agonist promotes nerve growth in vitro.
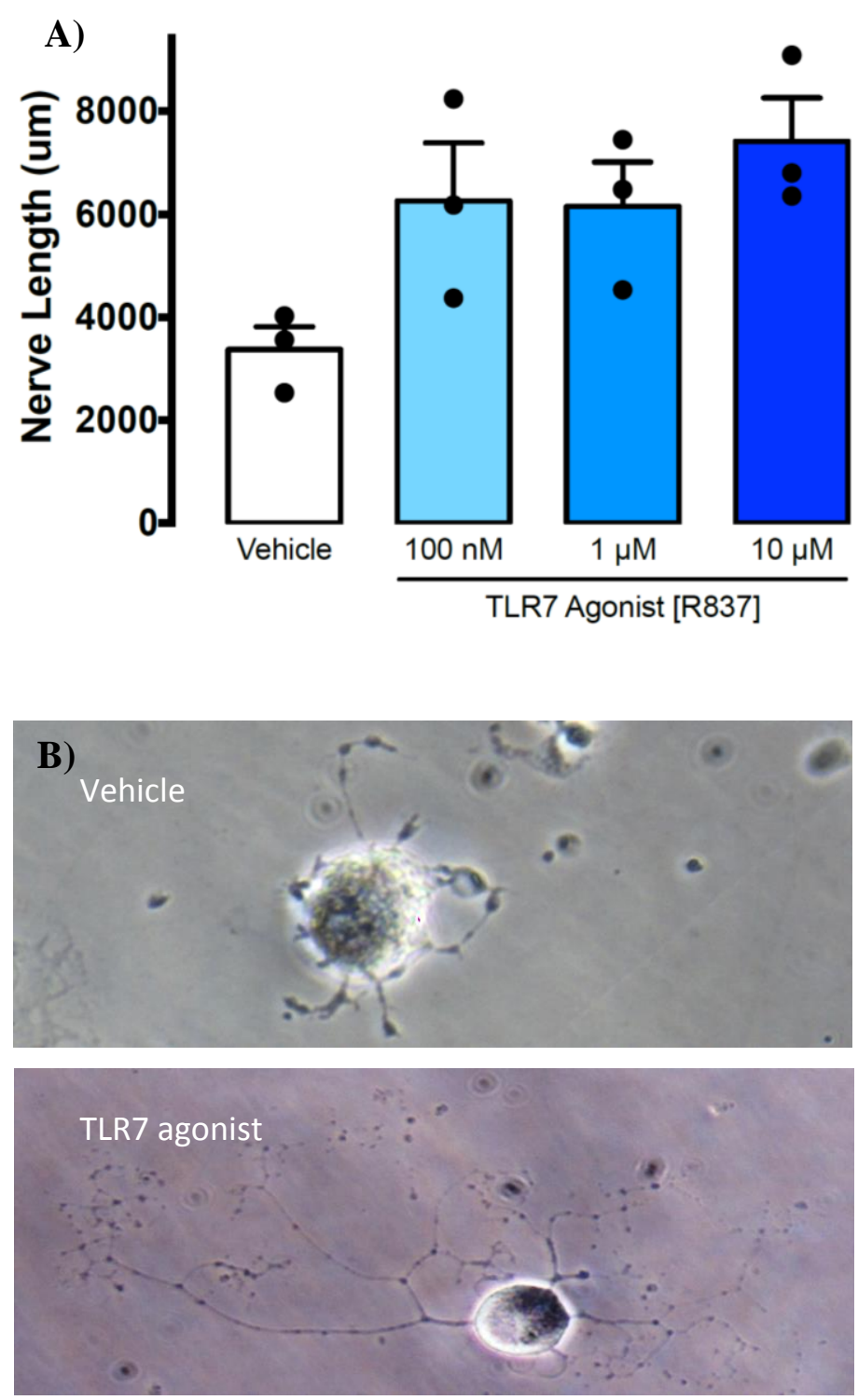

Figure 4. Guinea pig dorsal root ganglia were grown in culture were treated with the TLR7 agonist R837 (100nM-10 $\mu \mathrm{M})$ for 16 hours. A) Nerve length increased dose-dependently when stimulated with TLR7 agonist R837. B) Representative images of vehicle and TLR7 agonist-treated dorsal root ganglion $(10 \mu \mathrm{M})$. 


\section{DISCUSSION}

TLR7 is an immune receptor that detects respiratory virus and triggers an innate immune response including production of type 1 interferons and inflammatory cytokines (2). Previous studies have described TLR7 expression on airway sensory nerves (3). However, the specific subpopulation of sensory nerves expressing TLR7 has not been identified. In my study, I found TLR7 is primarily expressed by airway sensory $\mathrm{C}$ fibers, not sensory A $\delta$ fibers in guinea pig airways. Furthermore, TLR7 agonist increased sensory neurite length, suggesting TLR7 has a novel role regulating airway sensory nerve growth.

\section{Implications of TLR7 Expression by Sensory C fibers}

My findings provide evidence that TLR7 is predominantly expressed on airway sensory nerve C fibers. TLR7 expression on unmyelinated $\mathrm{C}$ fibers may be important for $\mathrm{C}$ fibers' control of cough and bronchoconstriction (17). For example, stimulating sensory $\mathrm{C}$ fiber afferents with irritants like capsaicin among other noxious stimuli (22) causes a central reflex leading to bronchoconstriction. Interestingly, previous studies have shown that TLR7 activation causes a rapid bronchodilation in the airways of guinea pigs in vivo and in vitro (28) and human airways in vitro (3). These contrasting results suggest that the $\mathrm{C}$ fibers are not exclusively involved in bronchoconstriction, but may also counter-balance constriction with relaxation mediated by TLR7. Furthermore, during periods of abnormal bronchoconstriction, such as during respiratory viral infections $(26,27)$, changes in TLR7 expression or function may contribute to abnormal regulation of airway tone.

TLR7 expression on C fibers also suggests that airway nerves can detect and respond to respiratory viruses (11). My findings suggest that within airway sensory nerves there are more TLR7-positive neurons than TLR7-negative neurons, and of these neurons, TLR7 is preferentially expressed on $\mathrm{C}$ fibers. These results contribute to the understanding of airway sensory $\mathrm{C}$ fibers as dynamic and integrated within complex immune and physiological responses due to their high expression of TLR7. 


\section{Implications of TLR7 regulation of sensory nerve growth}

This study also investigated the effect of a TLR7 agonist R837 (imiquimod) on nerve growth in vitro. Isolated guinea pig sensory nerves treated with R837 had increased nerve length and branching (data not shown). Increases in neurite length and branching of sensory neurons are linked to increased sensitivity to irritant-induced airway inflammation and hyperreactivity. Hyperinnervation of the airways amplifies specific responses to stimuli (22). In this study, TLR7 stimulation on airway sensory nerve $\mathrm{C}$ fibers promotes neurogenesis, potentially increasing the severity of responses to viral infections among other irritants, allergens, and pollutants. Given that TLR7 is activated by both R837 and respiratory viruses, the increased nerve growth (as a result of stimulation by the agonist), may demonstrate how respiratory viruses may worsen diseases like asthma by inducing changes to the nerve structure.

Prior studies have found that nerve growth can be induced from a variety of mediators including NGF and BDNF (19). Although I haven't identified the specific mechanism behind this dosedependent increase in neurite length, activation of TLR7 appears to be an underlying cause. The explanation for this may lie in TRL7's potent anti-viral response by the production of type 1 interferons and proinflammatory cytokines. In studies pertaining to imiquimod, a TLR7 agonist and the active component of a topical treatment called Aldara, application was noted to cause systemic pro-inflammatory cytokine production. In addition, protein levels of pro-inflammatory cytokines interleukin 1-beta and tumor necrosis factor alpha were significantly increased in the brain. (18). I hypothesized that elevated levels of pro-inflammatory cytokines have a downstream effect on neurotrophic factors initiating nerve growth.

Proinflammatory cytokines have a central role in the communication between the immune and nervous system. Under pathological conditions, overproduction of cytokines may detrimentally impact the nerve function. In particular, pro-inflammatory cytokines can adversely affect neurogenesis by decreasing brain-derived neurotrophic factor (29). Although the mechanism behind the observed nerve growth has yet to be explained, it is important to re-state that my findings demonstrate the neurotrophic effects of a TLR7 agonist on dorsal root ganglia in vitro. 
In sum, my study found TLR7 expression on C fibers of sensory afferent nerves and identified a novel neurotrophic effect of TLR7 stimulation on sensory nerves in vitro. Since respiratory viruses that activate TLR7 also exacerbate bronchoconstriction in lung diseases like asthma, my results provide compelling evidence that TLR7 expressed on sensory C fibers may contribute to aberrant bronchoconstriction and inflammation in this disease. Specifically, viruses may induce nerve growth by stimulating TLR7, leading to airways hyperreactivity and excessive bronchoconstriction. My findings contribute to the growing discussion of TLR7 as a therapeutic target for asthma due to its immunomodulatory capabilities and role in neuroplasticity. 


\section{REFERENCES}

1. Kawai T, Akira S. The roles of TLRs, RLRs and NLRs in pathogen recognition. Int Immunol. 2009;21:317-337.

2. Q. Sha, A.Q. Truong-Tran, J.R. Plitt, L.A. Beck, R.P. Schleimer. Activation of airway epithelial cells by toll-like receptor agonists. Am J Respir Cell Mol Biol. 2004;31:358-364.

3. Drake MG, Scott GD, Proskocil BJ, Fryer AD, Jacoby DB, Kaufman EH. Toll-like Receptor 7 Rapidly Relaxes Human Airways. Am J Respir Crit Care Med. 2013;188(6):664-672.

4. Schomburg ED, Steffens H, Mense S. Contribution of TTX-resistant C-fibres and A-fibres to nociceptive flexor-reflex and non-flexor-reflex pathways in cats. Neurosci Res. 2000;37:277-287.

5. Kimura S, Honda M, Tanabe M, Ono H. Noxious stimuli evoke a biphasic flexor reflex composed of A delta-fiber-mediated short-latency and C-fiber-mediated long-latency withdrawal movements in mice. J Pharmacol Sci. 2004;95(1):94-100.

6. Lawson SN, Waddell PJ. Soma neurofilament immunoreactivity is related to cell size and fibre conduction velocity in rat primary sensory neurons. J Physiol. 1991;435:41-63.

7. Lawson SN, Perry MJ, Prabhakar E, McCarthy PW. Primary sensory neurones: neurofilament, neuropeptides, and conduction velocity. Brain Res Bull. 1993;30(3-4):239-43.

8. Zakir HM, Mostafeezur RM, Suzuki A, Hitomi S, Suzuki I, Maeda T, Seo K, Yamada Y, Yamamura K, Lev S, Binshtok AM, Iwata K, Kitagawa J. Expression of TRPV1 channels after nerve injury provides an essential delivery tool for neuropathic pain attenuation. PLoS ONE. 2012;7(9):e44023. 
9. Jankowski MP, Soneji DJ, Ekmann KM, Anderson CE, Koerber HR. Dynamic changes in heat transducing channel TRPV1 expression regulate mechanically insensitive, heat sensitive C-fiber recruitment after axotomy and regeneration. J Neurosci. 2012; 32(49):17869-73.

10. Jeffery PK. Structural, immunologic, and neural elements in the normal human airway wall. Boston: Blackwell Scientific Publications.

11. Kaufman EH. Acute bronchodilator effects of toll-like receptor 7 agonists. Scholar Archive. $2011 ; 684$.

12. Lee LY, Kou YR, Frazier DT, Beck ER, Pisarri TE, Coleridge HM, Coleridge JC. Stimulation of vagal pulmonary C-fibers by a single breath of cigarette smoke in dogs. J Appl Physiol. 1989;66:2032-2038.

13. Coleridge HM, Coleridge JC. Pulmonary reflexes: neural mechanisms of pulmonary defense. Annu Rev Physiol. 1994;56:69-9.

14. Coleridge JC, Coleridge HM, Schelegle ES, Green JF. Acute inhalation of ozone stimulates bronchial C-fibers and rapidly adapting receptors in dogs. J Appl Physiol. 1993;74:2345-2352.

15. Giesbrecht GG, Pisarri TE, Coleridge JC, Coleridge HM. Cooling the pulmonary blood in dogs alters activity of pulmonary vagal afferents. J Appl Physiol. 1993;74:24-30.

16. Kalia MP. Organization of central control of airways. Annu Rev Physiol 1987;49:595-609.

17. Polverino M, Polverino F, Fasolino M, Andò F, Alfieri A, De Blasio F. Anatomy and neuropathophysiology of the cough reflex arc. Multidiscip Respir Med. 2012;7(1):5.doi:10.1186/20496958-7-5. 
18. Nerurkar L, McColl A, Graham G, Cavanagh J. The Systemic Response to Topical Aldara Treatment is Mediated Through Direct TLR7 Stimulation as Imiquimod Enters the Circulation. Sci Rep. 2017;7:16570. doi:10.1038/s41598-017-16707-5.

19. Kalil K, Dent EW. Branch management: mechanisms of axon branching in the developing vertebrate CNS. Nature. 2014;15:7-18.doi:10.1038/nrn3650.

20. Liem L. Stimulation of the Dorsal Root Ganglion. Prog Neurol Surg. 2015;29:213-224.doi: $10.1159 / 000434673$.

21. Brierley SM, Hughes P, Harrington A, Blackshaw AL. Chapter 24: Innervation of the Gastrointestinal Tract by Spinal and Vagal Afferent Nerves. In Johnson L, eds. Physiology of the Gastrointestinal Tract. $5^{\text {th }}$ ed. San Diego, CA. Elsevier; 2012: 703-731.

22. Hoyle GW, Graham RM, Finkelstein JB, Nguyen KPT, Gozal D, Friedman M. Hyperinnervation of the airways in transgenic mice overexpressing nerve-growth factor. Am $J$ Respir Cell Mol Biol. 1998;18:149-157.

23. Marshak-Rothstein A. Toll-like receptors in systemic autoimmune disease. Nat Rev Immunol. 2006;6(11):823-835.

24. Janeway CA, Medzhitov R. Innate immune recognition. Annu Rev Immunol. 2002;20:197-216.

25. Atmar RL, Guy E, Guntupalli KK, Zimmerman JL, Bandi VD, Baxter BD, Greenberg SB. Respiratory tract viral infections in inner-city asthmatic adults. Arch Intern Med. 1998;158:24532459.

26. Empey DW, Laitinen LA, Jacobs L, Gold WM, Nadel JA. Mechanisms of bronchial hyperreactivity in normal subjects after upper respiratory tract infection. Am Rev Respir Dis. 1976;113:131-139. 
27. Fryer AD, Jacoby DB. Parainfluenza virus infection damages inhibitory M2 muscarinic receptors on pulmonary parasympathetic nerves in the guinea-pig. $\mathrm{Br} \quad J$ Pharmacol. 1991;102:267-271.

28. Kaufman EH, Fryer AD, Jacoby DB. Toll-like receptor 7 agonists are potent and rapid bronchodilators in guinea pigs. J Allergy Clin Immunol. 2011;127:462-469.

29. Calabrese F, Rossetti AC, Racagni G, Gass P, Riva MA, Molteni R. Brain-derived neurotrophic factor: a bridge between inflammation and neuroplasticity. Front Cell Neurosci. 2014;8:430. doi:10.3389/fncel.2014.00430.

30. Sel S, Wegmann M, Sel S, Bauer S, Garn H, Alber G, Renz H. Immunomodulatory effects of viral TLR ligands on experimental asthma depend on the additive effects of IL-12 and IL-10. $J$ Immunol. 2007;178:7805-7813.

31. Kaufman EH, Jacoby DB. Upping the antedrug: is a novel anti-inflammatory Toll-like receptor 7 agonist also a bronchodilator? Br J Pharmacol. 2012;166:569-572.

32. Drake MG, Kaufman EH, Fryer AD, Jacoby DB. The Therapeutic Potential of Toll-like Receptor 7 Stimulation in Asthma. Inflamm Allergy Drug Targets. 2012;11(6):484-491.

33. Grace MS, Dubuis E, Birrell MA, Belvisi MG. Pre-clinical studies in cough research: Role of Transient Receptor Potential (TRP) channels. Pulm Pharmacol Ther. 2013; 26(5):498-507. 